\title{
ORIGINAL
} ARTICLES

\section{A Scoping Review of Trauma-Informed Curricula for Primary Care Providers}

\author{
Constance Gundacker, MD, MPH; Courtney Barry, PsyD, MS; Emily Laurent, MD; Rita Sieracki, MLS
}

BACKGROUND AND OBJECTIVES: Traumatic experiences such as abuse, neglect, and household dysfunction have a lifetime prevalence of $62 \%-75 \%$ and can negatively impact health outcomes. However, many primary care providers (PCPs) are inadequately prepared to treat patients with trauma due to a lack of training. Our objective was to identify trauma-informed approach curricula for PCPs, review their effectiveness, and identify gaps.

METHODS: We systematically identified articles from Medline, Scopus, Web of Science, Academic Search Premier, Cochrane, PsycINFO, MedEd Portal, and the STFM Resource Library. Search term headings "trauma-informed care (TIC)," "resilience," "patient-centered care," "primary care," and "education." Inclusion criteria were $\mathrm{PCP}$, pediatric and adult patients, and training evaluation. Exclusion criteria were outside the United States, non-English articles, non-PCPs, and inpatient settings. We used the TIC pyramid to extract topics. We analyzed evaluation methods using the Kirkpatrick Model.

RESULTS: Researchers reviewed 6,825 articles and identified 17 different curricula. Understanding health effects of trauma was the most common topic (94\%). Evaluation data revealed overall positive reactions and improved knowledge, attitudes, and confidence. Half (53\%) reported Kirkpatrick level 3 behavior change evaluation outcomes with increased trauma screening and communication, but no change in referrals. Only 12\% (2/17) evaluated Kirkpatrick level 4 patient satisfaction (significant results) and health outcomes (not significant).

CONCLUSIONS: Pilot findings from studies in our review show trauma-informed curricula for PCPs reveal positive reactions, an increase in knowledge, screening, communication, and patient satisfaction, but no change in referrals or health outcomes. Further research is needed to examine the impact of trainings on quality of care and health outcomes.

(Fam Med. 2021;53(10):843-56.)

doi: 10.22454/FamMed.2021.500263

W ith an increasingly higher prevalence $(62 \%-75 \%)$ of individuals experiencing a traumatic event, such as abuse, neglect, or household dysfunction (domestic violence, substance abuse, divorce, incarcerated relative, untreated mental illness) throughout their lives, trauma and care via a trauma-informed approach have become active areas of study. ${ }^{1-3} \mathrm{~A}$ trauma-informed approach is defined by the Substance Abuse and Mental Health Services Administration as

...a program, organization, or system that $[. .$.$] realizes the wide-$ spread impact of trauma and understands potential paths for recovery; recognizes the signs and symptoms of trauma in clients, families, staff, and others involved with the system; responds by fully integrating knowledge about trauma into policies, procedures, and practices; and seeks to actively resist re-traumatization. ${ }^{4}$

In the foundational "Adverse Childhood Experiences (ACE) Study" on childhood trauma, over half of the patients reported having at least one adverse childhood experience, including physical or psychological abuse and household dysfunction. ${ }^{1}$ These traumatic experiences can have lasting effects on survivors including increased health care utilization and expenditure, mental health problems such as posttraumatic stress disorder and anxiety, as well as cardiovascular and other physical diseases. ${ }^{1,5-9}$

Given the prevalence of trauma, it is likely primary care providers (PCPs) will interact with patients who have experienced trauma at some point during their lifetime. It is vital that PCPs are trained on trauma, its impact on health, and skills and strategies for implementing a trauma-informed approach, to provide high-quality care and improve health outcomes. Studies on the outcomes of implementing a trauma-informed approach in behavioral and mental health settings show that a trauma-informed approach improves patient care, provider satisfaction, and health outcomes by reducing depression, increasing compliance and

From the Department of Pediatrics (Dr Gundacker), and Departments of Psychiatry and Behavioral Medicine, and Family and Community Medicine (Dr Barry), Medical College of Wisconsin, Milwaukee, WI (Ms Sieracki); and Tulane University, New Orleans, LA (Dr Laurent). 
engagement, and improving provider understanding of patient needs. ${ }^{10,11}$ However, many PCPs currently feel discomfort in treating patients with prior traumatic experiences due to a lack of time, feeling uncomfortable, and unsure as to how to respond. ${ }^{12}$ Discomfort with discussing trauma and symptoms from prior traumatic experiences may hinder the patientprovider relationship and patient health outcomes. ${ }^{12,13}$ Patients with previous traumas are more likely to avoid health care preventative care visits and rely primarily on emergency room and urgent care visits for treatment, which ultimately results in increased morbidity and poorer outcomes throughout their lifespans. ${ }^{14,15}$

It is important that primary care providers are trained in working with patients with a trauma history to be able to meet their needs and help patients feel more comfortable going to preventative visits, rather than seeking emergency room care. One study of family medicine residency programs found that only about $27 \%$ of respondent programs reported including education and training on trauma-informed care (TIC). ${ }^{16}$ Of those who included TIC in their curriculum, $72.9 \%$ reported dedicating 5 or less hours per year to trauma education. While many family medicine residency programs did not have TIC curricula, family medicine residency program directors expressed a desire for the curricula to meet their patients' needs. TIC curricula for PCPs can help fill that gap.

Given the prevalence of trauma, the potentially negative impact it can have on health throughout the lifespan, and the fact that many PCPs do not feel prepared to work with patients with prior traumatic experiences, the objective of this scoping review was to identify and review the effectiveness of PCP trauma-informed approach curricula in the United States. Identifying current PCP trainings that utilize a trauma-informed approach will assist teaching programs, providers, and practices interested in improving knowledge, confidence, and skills in trauma-informed approaches. Furthermore, strengths and gaps in current trauma-informed approach curricula can be identified so that researchers and educators can adapt and develop curricula to improve patient care and outcomes.

\section{Methods}

The approach of this scoping review meets the guidelines for the PRISMA extension for scoping reviews (PRISMA-ScR) ${ }^{17}$ We selected articles for review using search term headings including "trauma-informed care," "resilience," "patient-centered care," "primary care," "education," and "training." We combined the search results for each heading to find articles related to trauma-informed approach curricula for primary care providers. The search strategy for Ovid Medline is shown in Table 1 . We identified articles by searching online databases including Ovid Medline, PsycINFO, Cochrane Library, Scopus, Web of Science, EBSCO's Academic Search Premier, and MedEd Portal. There was no restriction on year or status of publication; articles through August 25, 2020 were included in the search. We also searched the STFM Resource Library on February 22, 2021 using the terms "trauma informed care" and "adverse childhood experience." We considered grey literature, including conference abstracts, if they met inclusion and exclusion criteria. Article inclusion and exclusion criteria are shown in Table 2. We defined trauma using the Substance Abuse and Mental Health Services Administration's concept as it combines existing definitions and expert panel input:

Individual trauma results from (1) an event, series of events, or set of circumstances (2) that is experienced by an individual as physically or emotionally harmful or life threatening and (3) that has lasting adverse effects on the individual's function and mental, physical, social, emotional, or spiritual wellbeing. ${ }^{4}$
Two of the authors uploaded database search results to Rayyan for a blinded, independent evaluation. Rayyan is a web-based application that assists authors completing systematic reviews by providing a platform for article upload, blinded and unblinded comparison of article inclusion/exclusion by each contributing author, and monitoring of reasons for exclusion. ${ }^{18}$ Each article was screened by at least two authors and included or excluded based first on title/abstract review, and subsequently on full-text review. Following independent screening, any articles that were not unanimously included or excluded were discussed as a group to resolve any conflicts. Final analysis included identification of unique curricula from each of the articles.

We evaluated curricula based on the following criteria: participants, mode and length of training, TIC pyramid category, and evaluation methods and results. We utilized the TIC pyramid, a conceptual and operational framework developed by Raja et al, to extract data from the articles. ${ }^{19}$ This framework breaks down trauma into five categories: (1) patient-centered communication and care, (2) understanding the health effects of trauma, (3) interprofessional collaboration, (4) understanding your own history and reactions, and (5) screening. These categories fall under two main domains (universal trauma precautions and trauma-specific strategies). The first two categories are considered universal trauma precautions as they help establish trust/rapport and are applicable to all patients, regardless if trauma history is known or unknown. The last three categories are trauma-specific strategies and are used when a patient's trauma history is known. The description of the training topics covered in each curriculum were extracted under each of these five TIC pyramid categories (Table 3 , column 4). To assess change and outcomes, we utilized the Kirkpatrick Model focusing on different levels of learning as shown in Figure 1. ${ }^{20}$ 
Table 1: Ovid Medline Search Strategy, 1946 to August 25, 2020

1 (trauma informed or (aces or adverse child* event* or adverse child* experience*)).mp.

2 trauma.ti. or trauma.ab. or traumatiz*.mp. or traumatis*.mp.

3 exp stress, psychological/ or psychological stress*.mp. or stressful event*.mp. or stressful experience*.mp. or exp life change events/ or life chang* event*.mp.

4 exp Resilience, Psychological/ or resilien*.mp. or coping.mp. or cope.mp. or coped.mp.

5 exp Adaptation, Psychological/ or (psychological* adj5 adapt*).mp. or (emotional* adj5 adjust*).mp. or exp emotional adjustment/

6 exp Stress Disorders, Post-Traumatic/ or post traumatic stress disorder*.mp. or posttraumatic stress disorder*.mp. or ptsd.mp. or posttraumatic neuros*.mp. or post traumatic neuros*.mp. or (moral* adj5 injur*).mp.

7 exp social support/ or social support*.mp. or social network*.mp.

8 exp self care/ or self care.mp.

9 well being.mp. or exp "Quality of Life"/ or qol.mp. or quality of life.mp. or life quality.mp.

10 or/2-9

11 patient centered*.mp. or exp Patient-Centered Care/ or patient focused*.mp. or medical home*.mp. or client centered*.mp.

12 exp "Delivery of Health Care, Integrated"/ or (behavioral adj5 health adj5 integrat*).mp. or (behavioural adj5 health adj5 integrat*).mp. or (integrated adj5 care).mp.

1311 or 12

$14 \quad 10$ and 13

151 or 14

16 exp education/ or exp curriculum/ or exp education, professional/ or exp education, medical/or curricul*.mp. or ed.fs.

17 (educat* or train* or orientat* or lectur* or teach*).mp.

18 exp Lectures/ or exp simulation/ or simulat*.mp. or screen*.mp.

19 exp Teaching/ or exp Teaching Materials/

20 exp Education, Medical, Continuing/ or continuing medical educat*.mp. or cme.mp.

21 exp Health Personnel/ed or interprofessional educat*.mp.

22 exp program development/ or (program* adj5 develop*).mp.

23 exp quality improvement/ or (quality adj5 improv*).mp.

24 exp Evaluation Studies as Topic/ or (research adj5 evaluat*).mp. or (program* adj5 evaluat*).mp.

25 or/16-24

26 exp Primary Health Care/ or primary care.mp. or primary health care.mp.

27 exp Family Practice/ or family medicine.mp. or family practice.mp. or family practitioner*.mp.

28 exp Physicians, Family/ or family physician*.mp. or family doctor*.mp.

29 exp Pediatrics/ or (pediatric* or paediatric*).mp.

30 internal medicine.mp. or exp Internal Medicine/

31 or $/ 26-30$

$32 \quad 15$ and 25 and 31

33 limit 32 to english language

Table 2: Inclusion/Exclusion Criteria for Scoping Review of TraumaInformed Approach Curricula for Primary Care Providers

\begin{tabular}{|c|c|}
\hline Inclusion Criteria & Exclusion Criteria \\
\hline $\begin{array}{l}\text { Population = } \\
\text { - Primary care providers (medical doctors and advanced } \\
\text { practice providers) } \\
\text { - Primary care residents (family medicine, internal } \\
\text { medicine, pediatrics, combined medicine and pediatrics) }\end{array}$ & - Nonprimary care providers, including medical students \\
\hline $\begin{array}{l}\text { Concept }= \\
\text { - Trauma-informed approach training for primary care } \\
\text { providers of pediatric and/or adult patients } \\
\text { - Includes description of training evaluation (utilizing the } \\
\text { Kirkpatrick Model) }\end{array}$ & $\begin{array}{l}\text { - Guide for organizational implementation of trauma- } \\
\text { informed approach without describing provider training } \\
\text { - No description of training evaluation } \\
\text { - Adverse childhood experience or trauma screening with } \\
\text { no description of training }\end{array}$ \\
\hline $\begin{array}{l}\text { Context }= \\
\text { - Primary care outpatient setting } \\
\text { - In the United States } \\
\text { - English language }\end{array}$ & $\begin{array}{l}\text { - Inpatient and nonprimary care setting } \\
\text { - Outside the United States } \\
\text { - Non-English articles }\end{array}$ \\
\hline
\end{tabular}




\section{Results}

Database search identified 6,823 unique articles (Figure 2). We found two additional articles, one on final review of MedEd Portal on November 3, 2020, and another during the review process on February 22, 2021, for a total of 6,825 unique articles. After title and abstract review, we excluded 6,729 articles for relevance or non-US settings and the remaining 96 full-text articles were then reviewed independently by two authors for final inclusion or exclusion. Following the full-text review, 77 articles were excluded. The most common reasons for exclusion of articles at the full-text stage were no curricula or training and no evaluation of curricula. The resulting 19 articles, with two conference abstracts, ${ }^{21,22}$ were examined in-depth to determine the participants, mode and length of training, topics, and evaluation methods and results. ${ }^{21-39}$ We identified 17 different curricula in the 19 articles included for final analysis. The two articles by Green et al discuss the same curriculum, but evaluate different outcomes. ${ }^{23,24}$ Additionally, Wen et al and MillerCribbs discuss the same curriculum, but utilize different evaluations. ${ }^{30,39}$ Articles contained varying levels of description of training topics, mode and length of training, and evaluation, with some giving a brief mention of how primary care providers were trained as a small part of a larger manuscript, and others giving in-depth explanations as the focus of the manuscript (Table 3). Trainings were taught by a mixture of health professionals including TIC experts, clinical psychologists, social workers, physicians, and nurses. ${ }^{21-39}$

Table 3: Summary of 17 Trauma-Informed Approach Curricula for Primary Care Providers

\begin{tabular}{|c|c|c|c|c|c|}
\hline 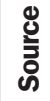 & Participants & $\begin{array}{l}\text { Mode, Length of } \\
\text { Training, CME }\end{array}$ & $\begin{array}{l}\text { TIC Pyramid } \\
\text { Category }\end{array}$ & Evaluation Method & Kirkpatrick Level and Results \\
\hline 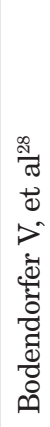 & $\begin{array}{l}\text { Primary care } \\
\text { physician } \\
\text { (PCPs; eight } \\
\text { residents, four } \\
\text { attendings, } \\
\text { one physician } \\
\text { assistant) } \\
\text { in family } \\
\text { medicine } \\
\text { residency } \\
\text { clinic }\end{array}$ & $\begin{array}{l}\text { Two sessions } \\
\text { (length not } \\
\text { specified) } \\
\text { including } \\
\text { discussion } \\
\text { on ACEs/ } \\
\text { health impacts, } \\
\text { workflow, sample } \\
\text { scripts, ACE } \\
\text { Conversation } \\
\text { card. }\end{array}$ & $\begin{array}{l}\text { - Patient-centered } \\
\text { communication } \\
\text { and care } \\
\text { - Understand the } \\
\text { health effects of } \\
\text { trauma } \\
\text { - Interprofessional } \\
\text { collaboration }\end{array}$ & $\begin{array}{l}\text { Postsurveys of: } \\
\text { (1) providers } \\
\text { on acceptability } \\
\text { and feasibility } \\
\text { (immediate and } \\
\text { quarterly x 4) and } \\
\text { (2) immediate } \\
\text { parent/guardian } \\
\text { (N=238) } \\
\text { acceptability. }\end{array}$ & $\begin{array}{l}\text { Kirkpatrick level } 1 \\
\text { 1. } 76 \% \text { parents/guardians felt positively } \\
\text { about ACE conversation. } \\
\text { 2. } 81 \% \text { parents/guardians felt } \\
\text { comfortable receiving information } \\
\text { from PCP. } \\
\text { 3. } 97 \% \text { parents/guardians preferred to } \\
\text { learn information from their PCP. } \\
\text { 4. } 71 \% \text { providers felt parents/guardians } \\
\text { receptive to ACE conversation. } \\
\text { 5. } 61 \% \text { providers said conversation took } \\
\text { 1-2 minutes. }\end{array}$ \\
\hline 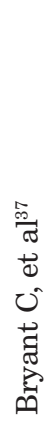 & $\begin{array}{l}59 \text { pediatric } \\
\text { PCPs (57 } \\
\text { medical } \\
\text { residents, } \\
\text { two nurse } \\
\text { practitioners) }\end{array}$ & $\begin{array}{l}\text { Education } \\
\text { as part of } \\
\text { QI initiative: } \\
\text { PowerPoint } \\
\text { presentation } \\
\text { (length not } \\
\text { specified). }\end{array}$ & $\begin{array}{l}\text { - Understand the } \\
\text { health effects of } \\
\text { trauma } \\
\text { - Interprofessional } \\
\text { collaboration } \\
\text { - Screening }\end{array}$ & Pre/posttest survey & $\begin{array}{l}\text { Kirkpatrick levels } 1,2 \& \text { 3: } \\
\text { 1. Statistically significant improvement } \\
\text { in provider's knowledge after ACE } \\
\text { education }(P<.001) \text { and awareness } \\
\text { of resources }(P<.001) \text {. } \\
\text { 2. Significant improvement in provider's } \\
\text { comfort level of screening }(P<.001) \text {. } \\
\text { 3. No change in provider's role of } \\
\text { screening for ACEs }(P=.13) \text {. } \\
\text { 4. Post-12 weeks implementation, } 480 \\
\quad \text { screening tools completed. } 93 \% \text { of } \\
\text { providers used tool. }\end{array}$ \\
\hline 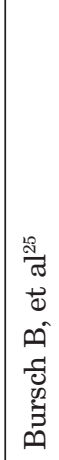 & $\begin{array}{l}\text { Pediatrics } \\
\text { and internal } \\
\text { medicine- } \\
\text { pediatrics } \\
\text { residents ( } 83 \\
\text { completed one } \\
\text { survey, } \\
27 \text { completed } \\
\text { both pre/ } \\
\text { postsurveys) }\end{array}$ & $\begin{array}{l}\text { Trauma- } \\
\text { informed, } \\
\text { evidence- } \\
\text { informed } \\
\text { resilience skills } \\
\text { training program. } \\
\text { Six 1-hour } \\
\text { modules with } \\
\text { biological/ } \\
\text { science frame, } \\
\text { skill-based } \\
\text { practice, and five- } \\
\text { 10-minute videos. }\end{array}$ & $\begin{array}{l}\text { - Patient-centered } \\
\text { communication } \\
\text { and care } \\
\text { - Understand the } \\
\text { health effects of } \\
\text { trauma } \\
\text { - Understand your } \\
\text { own history and } \\
\text { reactions }\end{array}$ & $\begin{array}{l}\text { Online pre/ } \\
\text { postsurvey on } \\
\text { beliefs and self- } \\
\text { efficacy. }\end{array}$ & $\begin{array}{l}\text { Kirkpatrick levels } 1 \& \mathbf{2} \text { : } \\
\text { 1. Increased self-perceived ability to } \\
\text { recognize trauma in others }(P=.005) \text {. } \\
\text { 2. Increased knowledge of evidence- } \\
\text { based approaches to assisting those } \\
\text { with trauma }(P<.001) \text {. } \\
\text { 3. Increased knowledge on what helps if } \\
\text { they disagree with medical decision } \\
\text { making of an attending with one of } \\
\text { their patients }(P=.01) \text {. } \\
\text { 4. More likely to believe attendings } \\
\text { affected by patient deaths }(P=.03) \text {. }\end{array}$ \\
\hline
\end{tabular}

(Continued on next page) 
Table 3: Continued

\begin{tabular}{|c|c|c|c|c|c|}
\hline 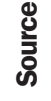 & Participants & $\begin{array}{l}\text { Mode, Length of } \\
\text { Training, CME }\end{array}$ & $\begin{array}{l}\text { TIC Pyramid } \\
\text { Category }\end{array}$ & Evaluation Method & Kirkpatrick Level and Results \\
\hline 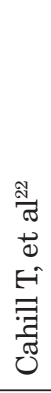 & $\begin{array}{l}\text { Residents } \\
\text { and faculty } \\
\text { at a family } \\
\text { medicine } \\
\text { residency } \\
\text { (number not } \\
\text { specified) }\end{array}$ & $\begin{array}{l}\text { Presentation } \\
\text { to residents } \\
\text { and faculty } \\
\text { on ACE study, } \\
\text { role play, ACE } \\
\text { questionnaire } \\
\text { given to patients } \\
\text { and education to } \\
\text { residents on how } \\
\text { to respond. }\end{array}$ & $\begin{array}{l}\text { - Patient-centered } \\
\text { communication } \\
\text { and care } \\
\text { - Understand the } \\
\text { health effects of } \\
\text { trauma } \\
\text { - Screening }\end{array}$ & $\begin{array}{l}\text { Qualitative } \\
\text { feedback from } \\
\text { residents, patients, } \\
\text { and faculty } \\
\text { including faculty } \\
\text { focus group. }\end{array}$ & $\begin{array}{l}\text { Kirkpatrick levels } 1 \text { \& } 2 \text { : } \\
\text { 1. All experiences with ACE } \\
\quad \text { intervention reported as positive. } \\
\text { 2. Recognized the importance of } \\
\quad \text { screening for mental and physical } \\
\text { health. } \\
\text { 3. Patients: No complaints or negative } \\
\text { interactions reported. } \\
\text { 4. Perceived increased resident } \\
\quad \text { confidence. }\end{array}$ \\
\hline 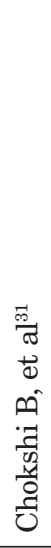 & $\begin{array}{l}28 \text { pediatric } \\
\text { residents, four } \\
\text { attending } \\
\text { physicians, } \\
\text { one fellow, } \\
\text { two medical } \\
\text { students }\end{array}$ & $\begin{array}{l}\text { Four computer- } \\
\text { based childhood } \\
\text { adversity } \\
\text { and trauma- } \\
\text { informed (CA- } \\
\text { TIC) individual, } \\
\text { interactive, } \\
\text { case-based } \\
\text { e-modules for } \\
\text { pediatric primary } \\
\text { care (2 hours). } \\
\text { Protected time to } \\
\text { complete during } \\
\text { 1-week advocacy } \\
\text { rotation. }\end{array}$ & $\begin{array}{l}\text { - Patient-centered } \\
\text { communication } \\
\text { and care } \\
\text { - Understand the } \\
\text { health effects of } \\
\text { trauma } \\
\text { - Interprofessional } \\
\text { collaboration } \\
\text { - Screening }\end{array}$ & $\begin{array}{l}\text { 17-item 5-point } \\
\text { Likert scale pre/ } \\
\text { postsession } \\
\text { questionnaire with } \\
\text { four categories } \\
\text { (knowledge, } \\
\text { attitudes, practice, } \\
\text { confidence). Post- } \\
\text { included three } \\
\text { short-answer } \\
\text { questions and } \\
\text { one Likert overall } \\
\text { rating question. }\end{array}$ & $\begin{array}{l}\text { Kirkpatrick levels } 1 \mathbf{\&} 2 \text { : } \\
\text { 1. Rated } 4.6 \text { out of } 5 \text { for design and } \\
\text { quality. } \\
\text { 2. Statistically significant increases } \\
\text { from pre- to postsession for } \\
\text { knowledge, attitudes, likelihood to } \\
\text { practice TIC, and confidence related } \\
\text { to CA-TIC }(P<.001) \text {. } \\
\text { 3. Most cited qualitative learning } \\
\text { points/practice changes: asking } \\
\text { about trauma in practice and } 7 \mathrm{C} \text { 's } \\
\text { of resilience. }\end{array}$ \\
\hline 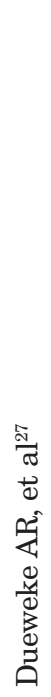 & $\begin{array}{l}33 \text { pediatric } \\
\text { residents }\end{array}$ & $\begin{array}{l}\text { One 2-hour in- } \\
\text { person } \\
\text { training. } \\
\text { Two-sided } \\
\text { index "pocket } \\
\text { card" with } \\
\text { information on } \\
\text { STAR acronym } \\
\text { (Screen/Tally/ } \\
\text { Ask additional } \\
\text { questions if } \\
\text { positive/Refer } \\
\text { if needed) and } \\
\text { local community } \\
\text { referral list. }\end{array}$ & $\begin{array}{l}\text { - Understand the } \\
\text { health effects of } \\
\text { trauma } \\
\text { - Interprofessional } \\
\text { collaboration } \\
\text { - Screening }\end{array}$ & $\begin{array}{l}\text { Posttraining } \\
\text { interviews } \\
\text { measured } \\
\text { perceptions. Pre/ } \\
\text { postsurveys on } \\
\text { attitudes, perceived } \\
\text { competence, } \\
\text { perceived barriers } \\
\text { to TIC practices. } \\
\text { Retrospective chart } \\
\text { review measured } \\
\text { pre/post change } \\
\text { in residents' TIC } \\
\text { practices (screen/ } \\
\text { refer). }\end{array}$ & $\begin{array}{l}\text { Kirkpatrick levels } 1, \mathbf{2} \& \mathbf{3} \text { : } \\
\text { 1. Training helpful; positive perceptions } \\
\text { STAR pocket card. } \\
\text { 2. Trend toward more favorable } \\
\text { attitudes }(P=.065) \text {; significantly } \\
\text { greater perceived competence } \\
\text { ( } P<.001) \text { to enact TIC practices; } \\
\text { decrease in perceptions that } \\
\text { time constraints, lack of training, } \\
\text { confusing information interfered } \\
\text { with provision of TIC practices at } \\
\text { T2 compared with T1 ( } P<.05) ; \text { no } \\
\text { change in perceptions that worry } \\
\text { about retraumatizing children/ } \\
\text { families interfered with provision of } \\
\text { TIC practices. } \\
\text { 3. Significant increase in completed } \\
\text { trauma screens }(P<.0001) \text {; slight } \\
\text { increase referrals for psychology/ } \\
\text { psychiatry services (not significant). }\end{array}$ \\
\hline
\end{tabular}


Table 3: Continued

\begin{tabular}{|c|c|c|c|c|c|}
\hline 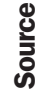 & Participants & $\begin{array}{l}\text { Mode, Length of } \\
\text { Training, CME }\end{array}$ & $\begin{array}{l}\text { TIC Pyramid } \\
\text { Category }\end{array}$ & Evaluation Method & Kirkpatrick Level and Results \\
\hline 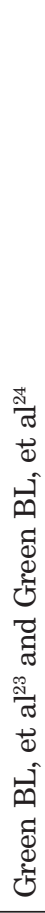 & $\begin{array}{l}17 \text { family } \\
\text { medicine } \\
\text { residents, } 13 \\
\text { community } \\
\text { physicians }\end{array}$ & $\begin{array}{l}\text { 6-hour course, } \\
\text { called Trauma- } \\
\text { Informed Medical } \\
\text { Care, delivered } \\
\text { in two sessions } \\
\text { at least } 1 \text { week } \\
\text { apart including: } \\
\text { case study, } \\
\text { experiential } \\
\text { exercises, poster, } \\
\text { worksheets, and } \\
\text { a manual. CME } \\
\text { offered. }\end{array}$ & $\begin{array}{l}\text { - Patient-centered } \\
\text { communication } \\
\text { and care } \\
\text { - Understand the } \\
\text { health effects of } \\
\text { trauma } \\
\text { - Understand your } \\
\text { own history and } \\
\text { reactions }\end{array}$ & $\begin{array}{l}\text { Randomized to } \\
\text { training or wait- } \\
\text { list. Patient- } \\
\text { centeredness score } \\
\text { derived from Roter } \\
\text { Interactional } \\
\text { Analysis System } \\
\text { ratings of three } \\
\text { audiotaped visits } \\
\text { of PCP and } \\
\text { standardized } \\
\text { patients. } \\
\text { Actual patients } \\
\text { completed surveys } \\
\text { before provider } \\
\text { trained or } 1 \text { week } \\
\text { to } 3 \text { months } \\
\text { after provider } \\
\text { trained (not } \\
\text { matched pre/post- } \\
\text {; overall numbers } \\
\text { compared). }\end{array}$ & $\begin{array}{l}\text { Kirkpatrick level } 3 \text { \& } 4 \text { : } \\
\text { Standardized Patients: } \\
\text { 1. Larger increase in patient } \\
\quad \text { centeredness score for immediately } \\
\text { trained PCPs compared with } \\
\text { delayed PCPs, moderate effect size } \\
\quad(0.66) \text {. } \\
\text { 2. Increased patient-centeredness } \\
\text { composite score for combined } \\
\text { trained PCPs pre- to posttraining } \\
\quad(P<.01) \text {. } \\
\text { Actual Patients: } \\
\text { 1. Increased Partnership score } \\
\text { posttraining }(P<.01) \text {. } \\
\text { 2. Increased Information score } \\
\text { posttraining (not statistically } \\
\text { significant, } P<.07) \text {. } \\
\text { 3. No difference in Rapport scale } \\
\text { posttraining. } \\
\text { 4. Patients with } \geq 1 \text { posttraumatic } \\
\text { stress disorder (PTSD) symptom } \\
(P<.01) \text { and patients with } \geq 2 \\
\text { traumas }(P=.02) \text { rated PCPs } \\
\text { lower on Partnership scale both } \\
\text { pre/posttraining compared with } \\
\text { patients without trauma or PTSD. }\end{array}$ \\
\hline 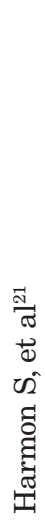 & $\begin{array}{l}35 \text { pediatric } \\
\text { residents }(26 \\
\text { completed } \\
\text { presurvey, } \\
17 \text { completed } \\
\text { postsurvey) }\end{array}$ & $\begin{array}{l}\text { Multidisciplinary } \\
\text { training: (1) } \\
\text { in-person } \\
\text { orientation, } \\
\text { (2) electronic } \\
\text { supplementary } \\
\text { materials, } \\
\text { (3) nurse } \\
\text { home visiting } \\
\text { experience. } \\
\text { Evidence-based } \\
\text { safety card to } \\
\text { guide trauma/ } \\
\text { resilience } \\
\text { discussions. }\end{array}$ & $\begin{array}{l}\text { - Understand the } \\
\text { health effects of } \\
\text { trauma } \\
\text { - Interprofessional } \\
\text { collaboration }\end{array}$ & $\begin{array}{l}\text { 13-item presurvey, } \\
\text { 18-item postsurvey. }\end{array}$ & $\begin{array}{l}\text { Kirkpatrick levels } 1 \text { \& } 2: \\
\text { 1. Increased understanding ( } 47 \% \text { vs. } \\
12 \% \text { strongly agree/agree post vs } \\
\text { pre) and comfort ( } 41 \text { vs } 0 \% \text { ) with } \\
\text { use of evidence-based safety card } \\
\text { intervention to discuss toxic stress } \\
\text { with families. } \\
\text { 2. Increased awareness of evidence- } \\
\text { based benefits of home visitation for } \\
\text { at-risk families ( } 59 \% \text { vs } 15 \% \text { ). } \\
\text { 3. Increased awareness of local home } \\
\text { visitation program ( } 71 \% \text { vs. } 23 \%) \text {. } \\
\text { 4. Greater confidence referring families } \\
\text { to home visitation programs ( } 35 \% \\
\text { vs } 7 \% \text { ). }\end{array}$ \\
\hline 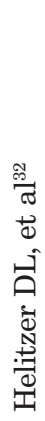 & $\begin{array}{l}26 \text { internal } \\
\text { medicine } \\
\text { and family } \\
\text { practice } \\
\text { providers } \\
\text { (physicians, } \\
\text { physician } \\
\text { assistants, } \\
\text { nurse } \\
\text { practitioners) }\end{array}$ & $\begin{array}{l}\text { Full day training } \\
\text { using lecture } \\
\text { and role-play. } \\
\text { Video-review } \\
\text { of simulated } \\
\text { patients and } \\
\text { audio review of } \\
\text { actual patient. } \\
\text { Two optional } \\
\text { role-playing } \\
\text { workshops. CME } \\
\text { offered. }\end{array}$ & $\begin{array}{l}\text { - Patient-centered } \\
\text { communication \& } \\
\text { care } \\
\text { - Understand the } \\
\text { health effects of } \\
\text { trauma } \\
\text { - Interprofessional } \\
\text { collaboration }\end{array}$ & $\begin{array}{l}\text { Randomized } \\
\text { Control Trial } \\
\text { 1) Simulated video- } \\
\text { taped patient } \\
\text { pre/post-full-day } \\
\text { training } \\
\text { 2) Actual patients: } \\
6 \text { months and } \\
18 \text { months } \\
\text { posttraining. }\end{array}$ & $\begin{array}{l}\text { Kirkpatrick level 3: } \\
\text { 1. Higher provider communication } \\
\text { proficiency score }(P<.05) \text {. Higher } \\
\text { patient-centeredness summary } \\
\text { score }(P<.05) \text {. } \\
\text { 2. 6-months posttraining: higher } \\
\text { patient-centeredness scores }(P<.01) \\
\text { and discussion of ACEs }(P<.001) . \\
\text { 18-months posttraining: higher } \\
\text { patient-centeredness summary } \\
\text { score }(P=.032) \text { and discussion of } \\
\text { ACEs }(P<.001) .\end{array}$ \\
\hline
\end{tabular}


Table 3: Continued

\begin{tabular}{|c|c|c|c|c|c|}
\hline 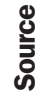 & Participants & $\begin{array}{l}\text { Mode, Length of } \\
\text { Training, CME }\end{array}$ & $\begin{array}{l}\text { TIC Pyramid } \\
\text { Category }\end{array}$ & Evaluation Method & Kirkpatrick Level and Results \\
\hline 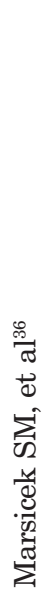 & $\begin{array}{l}\text { Pediatric } \\
\text { residents } \\
(\mathrm{n}=24 \\
\text { surveyed }) \text { and } \\
\text { faculty }(\mathrm{n}=5) .\end{array}$ & $\begin{array}{l}\text { Education as } \\
\text { part of quality } \\
\text { improvement } \\
\text { (QI) initiative for } \\
\text { ACEs screening: } \\
\text { 1-hr educational } \\
\text { seminar at } \\
1 \text { month; } \\
\text { simulation } \\
\text { experience of } \\
\text { at-risk ACE } \\
\text { score with } \\
\text { standardized } \\
\text { caregiver with } \\
\text { debriefing at } 2 \\
\text { months; lecture } \\
\text { at } 8 \text { months. }\end{array}$ & $\begin{array}{l}\text { - Understand the } \\
\text { health effects of } \\
\text { trauma } \\
\text { - Interprofessional } \\
\text { collaboration } \\
\text { - Screening }\end{array}$ & $\begin{array}{l}\text { Pre/postinitiative } \\
\text { surveys via email } \\
\text { (only resident } \\
\text { surveys compared } \\
\text { pre/post due to } \\
\text { insufficient faculty } \\
\text { numbers post). }\end{array}$ & $\begin{array}{l}\text { Kirkpatrick levels } 1 \text { \& } 2 \text { : } \\
\text { 1. No significant difference in resident } \\
\text { familiarity with clinical and } \\
\text { scientific findings of ACE study } \\
(P=.258) \text {. } \\
\text { 2. No significant difference in likelihood } \\
\text { to administer and assess an ACEs } \\
\text { questionnaire on patients }(P=.091) \text {. } \\
\text { 3. No significant difference in comfort } \\
\text { discussing patient's personal history } \\
\text { of physical, emotional, or sexual } \\
\text { abuse }(P=.219) \text {. } \\
\text { 4. No significant difference in } \\
\text { familiarity with local resources for } \\
\text { children exposed to ACEs }(P=.342) \text {. }\end{array}$ \\
\hline 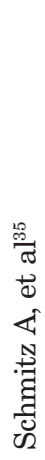 & $\begin{array}{l}\text { Pediatric } \\
\text { residents ( } 29 \\
\text { completed } \\
\text { premodule } \\
\text { survey, } 11 \\
\text { completed } \\
\text { pre/ } \\
\text { postsurvey) }\end{array}$ & $\begin{array}{l}25 \text {-minute self- } \\
\text { directed online } \\
\text { module. }\end{array}$ & $\begin{array}{l}\text { - Patient-centered } \\
\text { communication } \\
\text { and care } \\
\text { - Understand the } \\
\text { health effects of } \\
\text { trauma } \\
\text { - Understand your } \\
\text { own history and } \\
\text { reactions }\end{array}$ & $\begin{array}{l}\text { Pre/postsurvey } \\
\text { assessing: } \\
\text { 1) Knowledge of } \\
\text { ACEs, TIC, toxic } \\
\text { stress, resiliency } \\
\text { 2) Confidence in } \\
\text { discussing ACEs, } \\
\text { TIC, toxic stress, } \\
\text { resiliency } \\
\text { 3) Self-reported } \\
\text { frequency of } \\
\text { discussion of all } \\
\text { topics. }\end{array}$ & $\begin{array}{l}\text { Kirkpatrick levels } \mathbf{1}, \mathbf{2} \& \mathbf{3} \text { : } \\
\text { 1. Increase confidence in knowledge } \\
\text { of ACEs }(P<.05), \text { TIC }(P<.05), \\
\text { toxic stress }(P<.05) \text {, and resiliency } \\
\quad(P<.05) \text {. } \\
\text { 2. Increase in confidence in discussing } \\
\text { all topics }(\mathrm{ACEs}, \mathrm{TIC} \text {, toxic stress, } \\
\text { resilience }(P<.05) \text {. } \\
\text { 3. Increase in self-reported frequency of } \\
\quad \text { discussion of all topics }(P<.01) .\end{array}$ \\
\hline 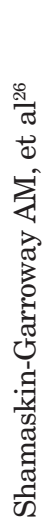 & $\begin{array}{l}\text { Eight medical } \\
\text { residents } \\
\text { (specialty not } \\
\text { specified) }\end{array}$ & $\begin{array}{l}\text { Three directly } \\
\text { observed patient } \\
\text { visits on } 3 \text { days } \\
\text { over } 2 \text {-week } \\
\text { VA rotation } \\
\text { at women's } \\
\text { primary care } \\
\text { clinic. Feedback } \\
\text { from clinical } \\
\text { psychology fellow. }\end{array}$ & $\begin{array}{l}\text { - Patient-centered } \\
\text { communication } \\
\text { and care }\end{array}$ & $\begin{array}{l}\text { Pre/posttraining } \\
\text { survey (Likert } \\
\text { scale) on self- } \\
\text { perceived skill, } \\
\text { comfort, use of } \\
\text { patient-centered } \\
\text { approaches, } \\
\text { understanding of } \\
\text { trauma-sensitive } \\
\text { care. }\end{array}$ & $\begin{array}{l}\text { Kirkpatrick levels } \mathbf{1}, \mathbf{2} \& \mathbf{3} \text { : } \\
\text { 1. Increased knowledge of strengths/ } \\
\text { weaknesses in interviewing } \\
\text { patients and confidence in patient } \\
\text { communication skills }(P=.03) \text {. } \\
\text { 2. Increased provider comfort discussing } \\
\text { trauma, knowledge of responding } \\
\text { sensitively to trauma exposure, } \\
\text { adjusting interview-style to TIC } \\
\text { ( } P=.01) \text {. } \\
\text { 3. No change in resident perception of } \\
\text { patient-centered interviewing skills, } \\
\text { comfort discussing mental health, } \\
\text { use of motivational interviewing } \\
\text { techniques. }\end{array}$ \\
\hline
\end{tabular}


Table 3: Continued

\begin{tabular}{|c|c|c|c|c|c|}
\hline 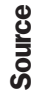 & Participants & $\begin{array}{l}\text { Mode, Length of } \\
\text { Training, CME }\end{array}$ & $\begin{array}{l}\text { TIC Pyramid } \\
\text { Category }\end{array}$ & Evaluation Method & Kirkpatrick Level and Results \\
\hline 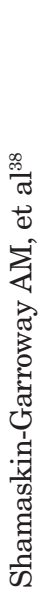 & $\begin{array}{l}16 \text { internal } \\
\text { medicine } \\
\text { residents, } \\
\text { five nurse } \\
\text { practitioner } \\
\text { residents at } \\
\text { a VA inter- } \\
\text { professional } \\
\text { primary care } \\
\text { residency } \\
\text { program }\end{array}$ & $\begin{array}{l}\text { Five 1-hour } \\
\text { didactic sessions; } \\
\text { 10-minute group } \\
\text { reflection rounds } \\
\text { regarding patient } \\
\text { interactions; } \\
\text { Optional patient } \\
\text { care observation } \\
\text { and feedback. }\end{array}$ & $\begin{array}{l}\text { - Patient-centered } \\
\text { communication } \\
\text { and care } \\
\text { - Understand the } \\
\text { health effects of } \\
\text { trauma } \\
\text { - Understand your } \\
\text { own history \& } \\
\text { reactions } \\
\text { - Screening }\end{array}$ & $\begin{array}{l}\text { Pre/post-self-report } \\
\text { questionnaire on } \\
\text { trauma-informed } \\
\text { primary care } \\
\text { (TIPC)-related } \\
\text { knowledge, } \\
\text { attitudes, beliefs, } \\
\text { and self-reported } \\
\text { practices. Feedback } \\
\text { on observed patient } \\
\text { encounters: } 10 \text {-item } \\
\text { checklist of specific } \\
\text { clinician behaviors } \\
\text { with written } \\
\text { quantitative } \\
\text { and qualitative } \\
\text { feedback. }\end{array}$ & $\begin{array}{l}\text { Kirkpatrick levels } \mathbf{1}, \mathbf{2} \& \mathbf{3} \text { : } \\
\text { 1. Improved self-reported knowledge } \\
\quad(P<.001) \text {. } \\
\text { 2. Improved self-reported trauma- } \\
\quad \text { informed attitudes }(P<.001) \text {. } \\
\text { 3. Improved self-reported trauma- } \\
\quad \text { informed practice }(P<.001) \text {. } \\
\text { 4. No significant change in beliefs about } \\
\quad \text { TIPC (however high baseline score). }\end{array}$ \\
\hline 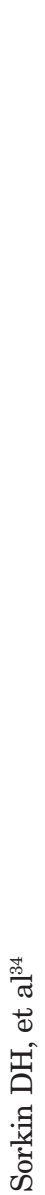 & $\begin{array}{l}16 \text { family } \\
\text { medicine } \\
\text { providers, } \\
\text { two general } \\
\text { internal } \\
\text { medicine } \\
\text { providers: } 10 \\
\text { intervention } \\
\text { arm/eight } \\
\text { control arm }\end{array}$ & $\begin{array}{l}\frac{\text { 3-Component }}{\text { Intervention Arm: }} \\
\begin{array}{l}\text { 1. One 3-hour } \\
\text { web-based } \\
\text { tutorial }\end{array} \\
\text { 2. Patient } \\
\text { screening } \\
\text { via iPad } \\
\text { 3. Web-based } \\
\text { mobile } \\
\text { application } \\
\text { with clinical } \\
\text { guidelines } \\
\text { and } \\
\text { algorithms } \\
\text { 2-Component } \\
\text { Control Arm: } \\
\text { 1. One online } \\
\text { tutorial } \\
\text { 2. Patient } \\
\text { screening }\end{array}$ & $\begin{array}{l}\text { - Patient-centered } \\
\text { communication } \\
\text { and care } \\
\text { - Understand the } \\
\text { health effects of } \\
\text { trauma } \\
\text { - Screening }\end{array}$ & $\begin{array}{l}\text { Baseline and 12- } \\
\text { week follow-up } \\
\text { assessment using } \\
\text { (1) electronic } \\
\text { mental health } \\
\text { screening tool, (2) } \\
\text { paper surveys, (3) } \\
\text { patient electronic/ } \\
\text { paper medical } \\
\text { record (initiation } \\
\text { of evidence- } \\
\text { based guideline } \\
\text { care, initiation of } \\
\text { trauma-informed } \\
\text { care as defined by } \\
\geq 1 \text { of following in } \\
\text { patient's records: } \\
\text { - conducted a risk } \\
\text { assessment of } \\
\text { depression/PTSD } \\
\text { - discussed trauma } \\
\text { history } \\
\text { - asked if patients } \\
\text { wanted to improve } \\
\text { their well-being } \\
\text { - assessed } \\
\text { psychiatric } \\
\text { symptoms). }\end{array}$ & $\begin{array}{l}\text { Kirkpatrick level } 3 \text { \& } 4 \text { : } \\
\text { 1. Intervention providers had } 4 \text { times } \\
\text { higher odds of utilizing evidence- } \\
\text { based guidance care }(P=.049) \text {. } \\
\text { 2. Intervention providers were more } \\
\text { likely to initiate medication } \\
\text { ( } P<.001) \text { or provide supportive } \\
\text { psychological counseling and } \\
\text { postpone prescription initiation } \\
\text { ( } P=.008) \text {. } \\
\text { 3. No difference in referral to mental } \\
\text { health }(P=.44) \text {. } \\
\text { 4. Intervention group had } 16 \text { times } \\
\text { higher odds of being provided with } \\
\text { trauma-informed care ( } P<.001) . \\
\text { 5. Identified with PTSD and depression } \\
\text { during screening } 5 \text { times higher } \\
\text { odds of receiving trauma informed } \\
\text { care compared with those who } \\
\text { were screened with depression only } \\
\text { ( } P=.001) \text {. } \\
\text { 6. Receiving evidence-based guideline } \\
\text { care significantly associated with } \\
\text { lower depression score at follow-up } \\
(P=.003) \text { but no significant changes } \\
\text { in PTSD scores. } \\
\text { 7. Receiving trauma-informed care } \\
\text { neither associated with changes } \\
\text { in depression nor PTSD scores at } \\
\text { follow-up. } \\
\text { 8. Intervention providers more likely } \\
\text { to diagnose depression }(P=.013) \\
\text { and PTSD ( } P=.002) \text { among those } \\
\text { screened. }\end{array}$ \\
\hline
\end{tabular}


Table 3: Continued

\begin{tabular}{|c|c|c|c|c|c|}
\hline ষ্ํㄹ & Participants & $\begin{array}{l}\text { Mode, Length of } \\
\text { Training, CME }\end{array}$ & $\begin{array}{l}\text { TIC Pyramid } \\
\text { Category }\end{array}$ & Evaluation Method & Kirkpatrick Level and Results \\
\hline 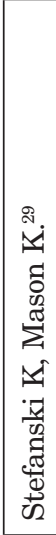 & $\begin{array}{l}18 \text { pediatric } \\
\text { residents }\end{array}$ & $\begin{array}{l}\underline{\text { Two-part }} \\
\text { Curriculum: } \\
\text { 1. Online } \\
\text { module } \\
\text { 2. Multimodal } \\
\text { in-person } \\
\text { workshop: } \\
\text { video } \\
\text { vignette, } \\
\text { didactics, } \\
\text { gamification } \\
\text { with } \\
\text { reflection, } \\
\text { facilitated } \\
\text { discussion. }\end{array}$ & $\begin{array}{l}\text { - Understand the } \\
\text { health effects of } \\
\text { trauma } \\
\text { - Screening }\end{array}$ & $\begin{array}{l}\text { Postworkshop } \\
\text { written feedback on } \\
\text { reaction and three } \\
\text { practice changes } \\
\text { plan to make. }\end{array}$ & $\begin{array}{l}\text { Kirkpatrick level 1: } \\
\text { 1. Residents most surprised by high } \\
\text { prevalence of ACEs reported in } \\
\text { initial ACE study. } \\
\text { 2. Perceived most helpful aspects of } \\
\text { trainings: interactive activities and } \\
\text { resources provided. } \\
\text { 3. Most common anticipated practice } \\
\text { changes was "more systematically } \\
\text { screening patients for ACEs". }\end{array}$ \\
\hline 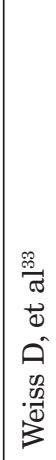 & $\begin{array}{l}440 \text { health } \\
\text { care } \\
\text { professionals } \\
\text { including } \\
\text { primary care } \\
\text { physicians } \\
\text { and residents } \\
\text { (specialty not } \\
\text { specified, } 294 \\
\text { completed } \\
\text { both pre/ } \\
\text { postsurveys) }\end{array}$ & $\begin{array}{l}\text { 1-hour in- } \\
\text { person session } \\
\text { using didactic } \\
\text { presentation and } \\
\text { group discussion. } \\
\text { CME offered. }\end{array}$ & $\begin{array}{l}\text { - Patient-centered } \\
\text { communication \& } \\
\text { care } \\
\text { - Understand the } \\
\text { health effects of } \\
\text { trauma } \\
\text { - Interprofessional } \\
\text { collaboration } \\
\text { - Understand your } \\
\text { own history and } \\
\text { reactions }\end{array}$ & $\begin{array}{l}\text { Pre/posttraining } \\
\text { Trauma-Informed } \\
\text { Medical Care } \\
\text { Questionnaire } \\
\text { (TIMCQ) and } \\
\text { Post-training } \\
\text { Satisfaction } \\
\text { Questionnaire. }\end{array}$ & $\begin{array}{l}\text { Kirkpatrick level } 1 \mathbf{\&} \mathbf{2 :} \\
\text { 1. Increased favorable attitude toward } \\
\text { TIC }(P<.001) \text {. } \\
\text { 2. Increased confidence in delivering } \\
\text { TIC }(P<.001) \text {. } \\
\text { 3. High satisfaction with trainings: } \\
90 \% \text { agreed/strongly agreed } \\
\text { acquired new knowledge/skills; } \\
\text { 86\% agreed/strongly agreed TIC } \\
\text { training enhanced their professional } \\
\text { expertise; } 88 \% \text { would recommend } \\
\text { the training. }\end{array}$ \\
\hline 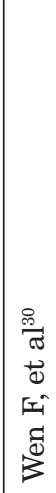 & $\begin{array}{l}\text { Internal } \\
\text { medicine } \\
\text { and family } \\
\text { medicine } \\
\text { residents } \\
\text { (59 in 2014- } \\
2015 \text { and } 22 \\
\text { preliminarily } \\
\text { in 2015-2016) }\end{array}$ & $\begin{array}{l}\text { Professional } \\
\text { ACEs-Informed } \\
\text { Training for } \\
\text { Health (PATH): } \\
\text { 4-hour training } \\
\text { with didactics, } \\
\text { provider and } \\
\text { patient videos, } \\
\text { standardized } \\
\text { patient (SP) } \\
\text { visits, trainee } \\
\text { feedback, } \\
\text { facilitated } \\
\text { discussion. }\end{array}$ & $\begin{array}{l}\text { - Patient-centered } \\
\text { communication } \\
\text { and care } \\
\text { - Understand the } \\
\text { health effects of } \\
\text { trauma } \\
\text { - Interprofessional } \\
\text { collaboration } \\
\text { - Understand your } \\
\text { own history and } \\
\text { reactions }\end{array}$ & $\begin{array}{l}\text { Online survey } 2-5 \\
\text { months following } \\
\text { the training. }\end{array}$ & $\begin{array}{l}\text { Kirkpatrick level 1, } \mathbf{2} \& \mathbf{3} \text { : } \\
\text { 1. Enhanced understanding of ACEs } \\
\text { (64.5\% initial, } 81.8 \% \text { 2015-2016 } \\
\text { preliminary results following } \\
\text { modifications to curriculum). } \\
\text { 2. Training helped apply concepts and } \\
\text { principles in practice (66\% initial, } \\
73 \% \text { revised). } \\
\text { 3. Faculty feedback was helpful ( } 77.4 \% \\
\text { initial, } 96 \% \text { revised). } \\
\text { 4. Planned on implementing the skills } \\
\text { learned in the simulation in clinical } \\
\text { practice ( } 62.5 \% \text { initial, } 63.6 \% \\
\text { revised). }\end{array}$ \\
\hline
\end{tabular}


Table 3: Continued

\begin{tabular}{|c|c|c|c|c|c|}
\hline 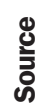 & Participants & $\begin{array}{l}\text { Mode, Length of } \\
\text { Training, CME }\end{array}$ & $\begin{array}{l}\text { TIC Pyramid } \\
\text { Category }\end{array}$ & Evaluation Method & Kirkpatrick Level and Results \\
\hline 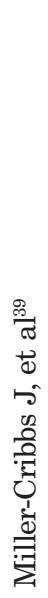 & $\begin{array}{l}53 \\
\text { participants } \\
\text { (38 family } \\
\text { medicine } \\
\text { or internal } \\
\text { medicine } \\
\text { residents } \\
\text { in years } 1 \\
\text { through } 4 \text {; } \\
15 \text { OT and } \\
\text { PT doctoral } \\
\text { students) }\end{array}$ & $\begin{array}{l}\text { Same PATH } \\
\text { curriculum as } \\
\text { above. Participate } \\
\text { each year of } \\
\text { residency: year } \\
1 \text { and } 2 \text { focus } \\
\text { on individual } \\
\text { ambulatory } \\
\text { encounters, year } \\
3 \text { focuses on } \\
\text { modifying the } \\
\text { SP ambulatory } \\
\text { encounter to } \\
\text { group simulation } \\
\text { for vicarious } \\
\text { learning. }\end{array}$ & Same as above & $\begin{array}{l}\text { Audiovisual } \\
\text { recordings of } \\
\text { individual learner- } \\
\text { standardized } \\
\text { patient encounters } \\
\text { coded using } \\
\text { standardized } \\
\text { behavioral } \\
\text { codes. Subset } 10 \\
\text { recordings of PC } \\
\text { residents who } \\
\text { participated in } \\
\text { simulations in first } \\
\text { and fourth years } \\
\text { of training allowed } \\
\text { for evaluation over } \\
\text { time. }\end{array}$ & $\begin{array}{l}\text { Kirkpatrick level 3: } \\
\text { Demonstrated skills congruent with TI } \\
\text { training on: } \\
\text { 1. Explaining ACEs } \\
\text { 2. Demonstrating empathy } \\
\text { 3. Collaborative treatment planning } \\
\text { 4. Stigma reduction } \\
\text { Year } 1 \text { compared with year 4: } \\
\text { 1. Slight increases in using metaphors } \\
\text { and infographics, explaining ACEs } \\
\text { effects on brain/body, stigma } \\
\text { reduction and validation } \\
\text { 2. Slight decreases in empathy and } \\
\text { collaborative treatment planning. }\end{array}$ \\
\hline
\end{tabular}

Abbreviations: CME, continuing medical education; PCP, primary care physician; ACE, adverse childhood experience; TI, trauma informed; TIC, trauma-informed care; TIPC, trauma-informed primary care; PTSD, posttraumatic stress disorder; SP, standardized patient.

Figure 1: Articles Reporting Curriculum Evaluation by Kirkpatrick Model Level of Learning ${ }^{20}$

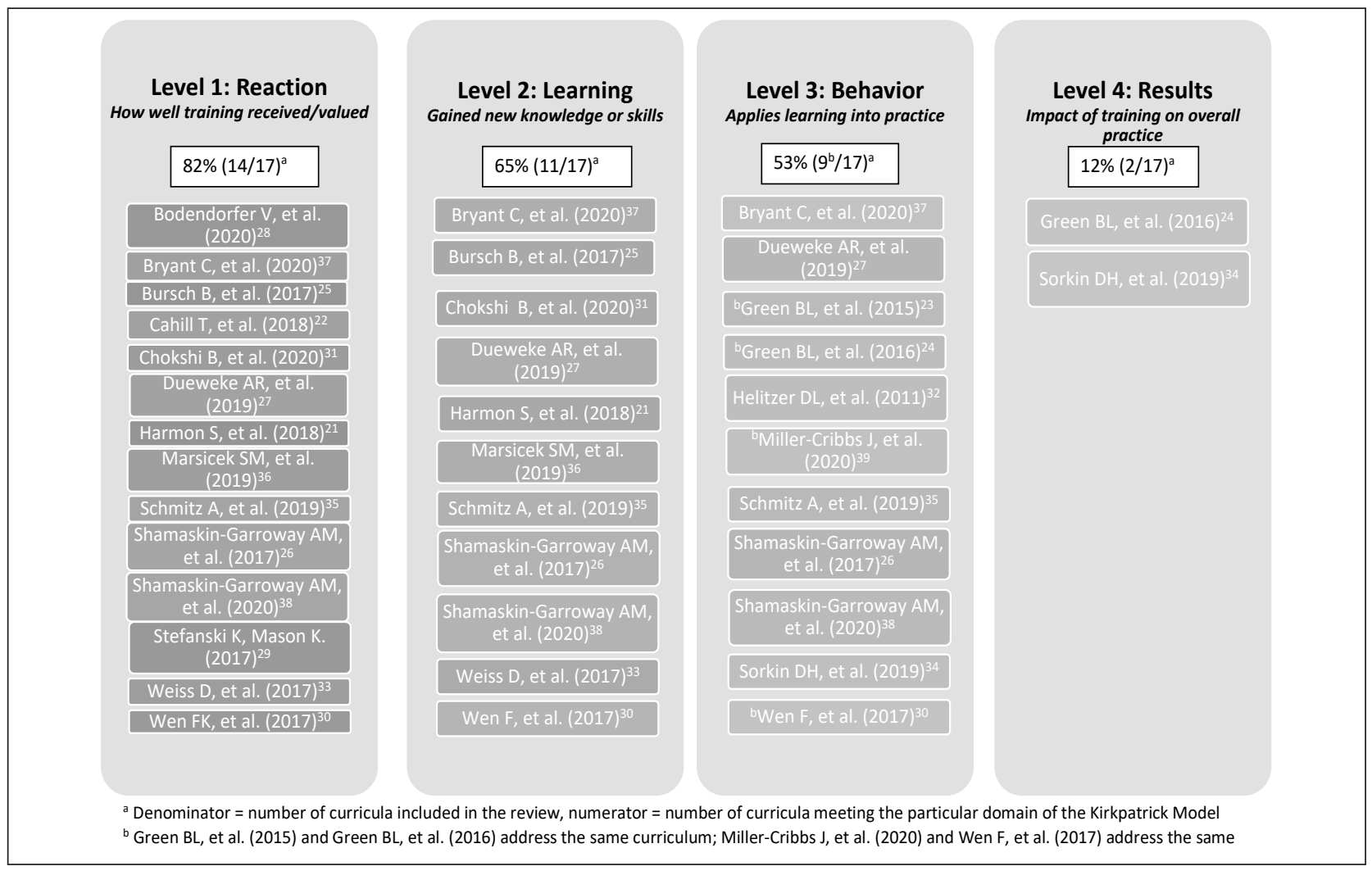


Figure 2: Source Selection Process

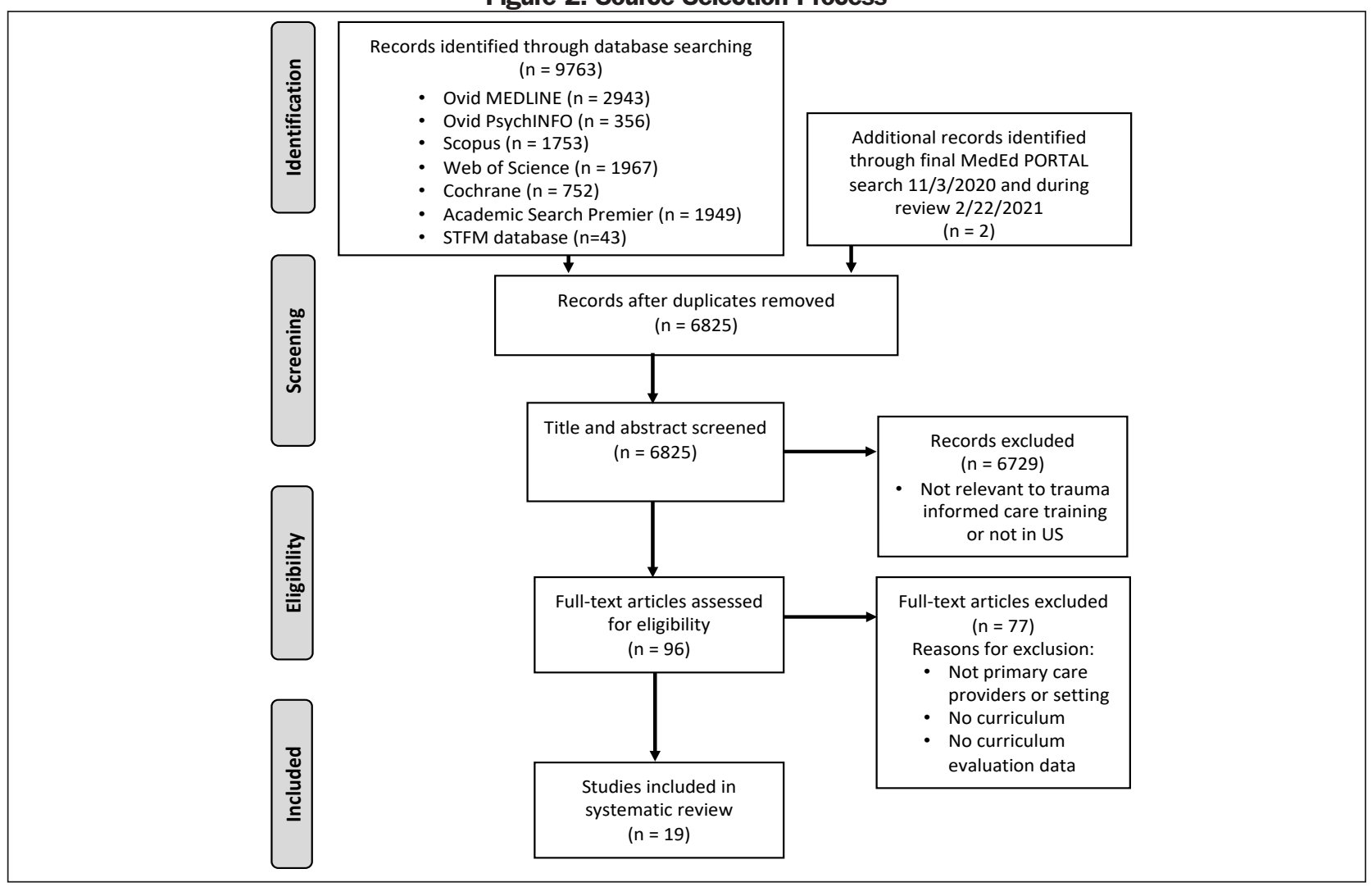

Half of the curricula self-identified as pilots, feasibility studies, or discussed curricula in development, revealing that trauma-related trainings for primary care providers is still an area of active development and research. ${ }^{23-31}$ Two educational curricula were adapted from prior curricula, while the remainder were created from a variety of sources, including Substance Abuse and Mental Health Services Administration principles, ${ }^{4}$ the American Academy of Pediatrics Trauma Toolbox for Primary Care,${ }^{40}$ guidelines from the American Psychological Association, ${ }^{41}$ evidence-based literature, and expert feedback. ${ }^{21-39}$ Bursch et al adapted the "Families OverComing Under Stress" resiliency training program (a strength-based, trauma-informed training program for deployed military personnel) for use with medical residents..$^{25}$ Green et al adapted the Risking Connection curriculum (a strength-based training program for helping people who have been hurt in interpersonal relationships, previously used in mental health, congregate care, faith-based, and medical facilities) for use with primary care residents, advanced practice providers, and physicians. ${ }^{23,24}$

Utilizing the TIC pyramid by Raja et al 2015 as a framework to extract TIC training topics from articles, ${ }^{19}$ the majority of the curricula covered topics related to universal trauma precautions; $71 \%$ (12/17) covered topics related to patient-centered communication and care ${ }^{22-26,28,30-35,38,39}$ and 94\% (16/17) covered topics related to understanding the health effects of trauma. ${ }^{21-25,27-38}$ The only curriculum that did not cover topics related to understanding the health effects of trauma was Shamaskin-Garroway et al, which focused on patient-centered communication skills. ${ }^{26}$ ShamaskinGarroway et al expanded on their initial pilot to include background teaching about trauma in their extended trauma-informed primary care curriculum. ${ }^{38}$ Patient-centered communication and care included patient-centered communication skills, trauma-related communication skills, communicating with patients with complex needs, and motivational interviewing. Many of the communication skills trainings discussed the importance of using alternative language and reframing negative comments, with a focus on patients who had experienced prior trauma. Curricula coverage of trauma-specific strategies included 53\% (9/17) addressing interprofessional collaboration ${ }^{21,27,28,30-33,36,37} ; 35 \%$ (6/17) addressing understanding your own history and reactions, ${ }^{23-25,30,33,35,38}$ and $47 \%$ (8/17) addressing screening. ${ }^{22,27,29,31,34,36-38}$

We analyzed reported evaluation methods using the Kirkpatrick Model's four levels of training evaluation as shown in Table 3 and Figure $1 .^{20}$ Examples of level 1 (reaction) evaluation results include training satisfaction, relevance, and engagement. ${ }^{21,22,25-31,33,35-38}$ Examples of level 2 (learning) evaluation results include increased favorable attitude toward TIC, and improved provider's 
knowledge after $\mathrm{ACE}$ education and awareness of resources..$^{21,25,31,33,37} \mathrm{We}$ evaluated behavior change (level 3) in a variety of ways including pre/ posttrainings chart review ${ }^{27,34}$; rated observed, audio or videotaped visits with patients (standardized or actual) $)^{23,24,32,38}$; patient surveys $\mathbf{s}^{24}$; and selfreported behavior change. ${ }^{26,35,38}$ Two studies reported level 4 outcomes for patient satisfaction and health outcome. Green et al had patients rate their PCPs using a survey derived from measures assessing patient satisfaction and interactions with providers. ${ }^{24}$ They found increased partnership scores posttraining, an increased but not significant information score, and no difference in rapport scores posttraining (excellent at baseline). Sorkin et al found receiving TIC was neither associated with changes in patients' depression nor posttraumatic stress disorder scores at 12 -week follow-up. ${ }^{34}$ Zero studies assessed impact of trainings on the organization (eg, cost).

\section{Discussion}

This review illustrates the relative infancy but growing literature base on the effectiveness of trauma-informed approach curricula for PCPs. As many of these curricula had relatively small sample sizes, their significance is limited, but may be promising, once tested in larger and multiple settings. Replicating, testing, and building on some of these curricula will advance the field further, rather than reinventing new curricula. Adaptations and additions can be made where current gaps exist in the training of PCPs. As training programs and primary care practices consider different traumainformed curricula for adoption and/ or adaptation in their program, the authors recommend the following considerations to guide curricula selection: (1) program goals and objectives, including patient population, training time availability, and setting; (2) the TIC pyramid categories (categories currently taught versus gaps); and (3) the desired Kirkpatrick Model level for evaluation.
If programs and practices currently have no TIC education, studies that have well-developed and explained curricula and/or modules include Bursch et al, ${ }^{25}$ Chokshi et al, ${ }^{31}$ Green et al, ${ }^{23,24}$ Schmitz, ${ }^{35}$ Sorkin et $a l,{ }^{34}$ Wen et al, ${ }^{30}$ and Miller-Cribs et al. ${ }^{39}$ Selection of which curricula to adopt/adapt should be guided by program needs. For example, if a practice/program is interested in improving skills training related to resiliency and vicarious trauma, we would recommend the curriculum used by Bursch et al. ${ }^{25}$ Online modules targeted for pediatric primary care providers are available for download via MedEdPortal for both Chokshi et $\mathrm{al}^{31}$ and Schmitz et al..$^{35}$ However, they have different TIC pyramid categories, time commitment, and delivery which might impact selection based on program/ practice need. The curricula described in Green et $\mathrm{al}^{23,24}$ and Wen et $\mathrm{al}^{30} /$ Miller-Cribs et al ${ }^{39}$ target adult primary care providers. Sorkin et $\mathrm{al}^{34}$ specifically focuses on a refugee population. While each of these curricula could be adaptable for other populations, it might be easiest for programs/practices to start with a previously developed curriculum aimed at their target population. Surveys previously used for evaluation can also be used, such as those used by Weiss et al ${ }^{33}$ (Trauma-Informed Medical Care Questionnaire) and Shamaskin-Garroway et $\mathrm{al}^{38}$ (Trauma-Informed Primary Care-related Knowledge, Attitudes, Beliefs and Self-reported Practices). Previously developed standardized primary care patient cases, such as those created by Helitzer et $\mathrm{al},{ }^{32}$ Green et $\mathrm{al}^{23}{ }^{23}$ and Wen et $\mathrm{al}^{30} / \mathrm{Miller}$ Cribs et al, ${ }^{39}$ can also be utilized in future studies to evaluate training effectiveness.

Educational topics and methods varied among the curricula, likely reflecting the needs and scheduling flexibility of the population trained (eg, residents vs practicing community physicians). Understanding health effects of trauma was the most common topic (94\%) utilizing a variety of modalities, including online, in-person, and a hybrid approach. Further research is needed to determine what elements of online versus inperson trainings are most beneficial. Furthermore, in identifying educational components, tangible resources for providers to refer to during the clinical encounter should be considered as a curriculum component and was used in multiple studies. ${ }^{21,27,28 \text {, }}$ ${ }^{34}$ Two of these studies measured objective behavior change through utilizing a pocket card ${ }^{27}$ and webbased mobile application..$^{34}$ Another component to consider for providers is three curricula offered continuing medical education credit for the training, which we believe is essential to incentivize practicing PCPs to participate in the training. ${ }^{23,24,32,33}$ Additionally, the two small randomized controlled trials that revealed a statistically significant improvement in provider communication scores used case studies, experiential exercises, or role plays. ${ }^{23,24,32} \mathrm{We}$ believe the use of interactive educational methods such as these is essential to allow providers to practice trauma-informed communication skills prior to interaction with actual patients. We were not able to determine a minimum curriculum coverage or time commitment related to evaluation outcome due to the pilot nature and heterogeneity of these studies. More research is needed to help answer this question, including how often booster or continuing training is needed.

Further research is needed to identify how training PCPs in delivering care via a trauma-informed approach can impact patient satisfaction, health outcomes, and cost of care. Only two studies evaluated Kirkpatrick level 4 patient outcome results: one with no change in patients' depression nor posttraumatic stress disorder scores at 12 -week follow-up ${ }^{34}$; and the other with patient's rating an increased partnership score posttraining, an increased but not significant information score, and no difference in rapport score posttraining (excellent at baseline). ${ }^{24}$ 
Zero studies evaluated impact of the training on the organization (eg, cost-effectiveness).

Additionally, further study is needed on the impact of trauma-informed approach curricula on providers' ability to recognize and respond to vicarious trauma (trauma symptoms that can result in providers from repeated exposure to other people's trauma). While at least three of the identified curricula included topics on vicarious trauma, self-awareness, and self-care, ${ }^{23-25,33}$ only one paper, that reported a small sample size, indicated measuring resiliency beliefs and self-efficacy, which showed a shift in the desired direction on several measures..$^{25}$ While it is important to identify trauma in patients and utilize a trauma-informed approach in treating these patients, it is also important to recognize how providers may respond to patients with a trauma history (countertransference) and the signs of secondary traumatic stress in providers in order to reduce burnout. In order to prevent secondary traumatic stress and ultimately burnout, more research is needed to further identify vicarious trauma for primary care providers and how addressing this type of trauma may impact burnout.

Limitations of this scoping review include the potential for publication bias and inclusion of only English articles published in the United States. It is possible other curricula exist that have been evaluated, but the curricula or the results may not have been published due to negative findings. However, multiple curricula identified in this review included both statistically significant and nonsignificant findings, showing evidence against publication bias. In this literature review, we chose to include only curricula that included an evaluation component. We did this to help limit the number of possible missed curricula as there are multiple curricula that have been, or are currently being developed, for which no evaluation data exist yet. Also, it is possible curricula exist in other countries and other languages, which were excluded for the purposes of this scoping review.

\section{Conclusion}

Current data on the impact of trauma-informed approach curricula for primary care providers reveals improved confidence in delivering care via a trauma-informed approach, improved knowledge about trauma and its impact on health outcomes, improved attitudes toward patients who have experienced prior trauma, and changes in PCP behavior posttraining with both simulated and actual patients. However, most of the current evidence is based on small pilot studies. Training programs and practices can adopt and/or adapt some of these curricula, utilizing the TIC pyramid framework and Kirkpatrick levels, based on their program's needs. Further research is needed to build improved referral connections to trauma-informed providers, and to examine how trauma-informed trainings might impact both short- and long-term quality of care, patient satisfaction, vicarious trauma, and health outcomes.

FINANCIAL SUPPORT: This project was supported by the Health Resources and Services Administration (HRSA) of the US Department of Health and Human Services (HHS) under grant number T32HP10030. This information or content and conclusions are those of the authors and should not be construed as the official position or policy of, nor should any endorsements be inferred by HRSA, HHS or the US Government.

PRESENTATIONS: This study was presented at the Medical College of Wisconsin's Department of Family and Community Medicine Research Poster Session, June 2018, Milwaukee, WI; at the North American Primary Care Research Group meeting, November 2018, Chicago, IL; and at the 40th Forum for Behavioral Science in Family Medicine, September 2019, Chicago, IL.

CORRESPONDING AUTHOR: Address correspondence to Dr Constance Gundacker, Department of Pediatrics, Medical College of Wisconsin, Children's Corporate Center, Suite C730, 999 N. 92nd Street, Milwaukee, WI 53226. 414-337-3264. Fax: 414-337-7105. cgundacker@mcw.edu.

\section{References}

1. Felitti VJ, Anda RF, Nordenberg D, et al. Relationship of childhood abuse and household dysfunction to many of the leading causes of death in adults. The Adverse Childhood Experiences (ACE) Study. Am J Prev Med. 1998;14(4):245258. doi:10.1016/S0749-3797(98)00017-8

2. Merrick MT, Ford DC, Ports KA, Guinn AS. Prevalence of adverse childhood experiences from the 2011-2014 Behavioral Risk Factor Surveillance System in 23 States. JAMA Pediatr. 2018;172(11):1038-1044. doi:10.1001/ jamapediatrics.2018.2537

3. Cronholm PF, Forke CM, Wade R, et al. Adverse childhood experiences: expanding the concept of adversity. Am J Prev Med. 2015;49(3):354361. doi:10.1016/j.amepre.2015.02.001

4. Substance Abuse and Mental Health Services Administration. SAMHSA's Concept of Trauma and Guidance for a Trauma-Informed Approach. HHS Publication No. (SMA) 14-4884. Rockville, MD: Substance Abuse and Mental Health Services Administration; 2014.

5. Nöthling J, Malan-Müller S, Abrahams N, Hemmings SMJ, Seedat S. Epigenetic alterations associated with childhood trauma and adult mental health outcomes: A systematic review. World J Biol Psychiatry. 2020;21(7):493512. doi:10.1080/15622975.2019.1583369

6. Bellis MA, Hughes K, Ford K, Ramos Rodriguez G, Sethi D, Passmore J. Life course health consequences and associated annual costs of adverse childhood experiences across Europe and North America: a systematic review and meta-analysis. Lancet Public Health. 2019;4(10):e517-e528. doi:10.1016/S24682667(19)30145-8

7. Petruccelli K, Davis J, Berman T. Adverse childhood experiences and associated health outcomes: A systematic review and metaanalysis. Child Abuse Negl. 2019;97:104127. doi:10.1016/j.chiabu.2019.104127

8. Kalmakis KA, Chiodo LM, Kent N, Meyer JS. Adverse childhood experiences, post-traumatic stress disorder symptoms, and self-reported stress among traditional and nontraditional college students. J Am Coll Health. 2020;68(4):411-418. doi:10.1080/07448481.20 19.1577860

9. Copeland WE, Shanahan L, Hinesley J, et al. Association of childhood trauma exposure with adult psychiatric disorders and functional outcomes. JAMA Netw Open. 2018;1(7):e184493. doi:10.1001/jamanetworkopen.2018.4493

10. Suarez E, Jackson DS, Slavin LA, Michels MS, McGeehan KM. Project Kealahou: improving Hawai'i's system of care for at-risk girls and young women through gender-responsive, trauma-informed care. Hawaii J Med Public Health. 2014;73(12):387-392

11. Hales TW, Green SA, Bissonette S, et al. Trauma-informed care outcome study. Res Soc Work Pract. 2019;29(5):529-539. doi:10.1177/1049731518766618

12. Palmieri J, Valentine JL. Using traumainformed care to address sexual assault and intimate partner violence in primary care. $\mathrm{J}$ Nurse Pract. 2021;17(1):44-48. doi:10.1016/j. nurpra.2020.08.028 
13. Green BL, Kaltman SI, Chung JY, Holt MP, Jackson S, Dozier M. Attachment and health care relationships in low-income women with trauma histories: a qualitative study. J Trauma Dissociation. 2012;13(2):190-208. doi:10.1080/1 5299732.2012.642761

14. Koball AM, Rasmussen C, Olson-Dorff D, Klevan J, Ramirez L, Domoff SE. The relationship between adverse childhood experiences, healthcare utilization, cost of care and medical comorbidities. Child Abuse Negl. 2019;90:120126. doi:10.1016/j.chiabu.2019.01.021

15. Hargreaves MK, Mouton CP, Liu J, Zhou YE, Blot WJ. Adverse childhood experiences and health care utilization in a low-income population. J Health Care Poor Underserved. 2019;30(2):749-767. doi:10.1353/hpu.2019.0054

16. Dichter ME, Teitelman A, Klusaritz H, Maurer DM, Cronholm PF, Doubeni CA. Traumainformed care training in family medicine residency programs results from a CERA survey. Fam Med. 2018;50(8):617-622. doi:10.22454/ FamMed.2018.505481

17. Tricco AC, Lillie E, Zarin W, et al. PRISMA extension for scoping reviews (PRISMA-ScR): checklist and explanation. Ann Intern Med. 2018;169(7):467-473. doi:10.7326/M18-0850

18. Ouzzani M, Hammady H, Fedorowicz Z, Elmagarmid A. Rayyan-a web and mobile app for systematic reviews. Syst Rev. 2016;5(1):210. doi:10.1186/s13643-016-0384-4

19. Raja S, Hasnain M, Hoersch M, Gove-Yin S, Rajagopalan C. Trauma informed care in medicine: current knowledge and future research directions. Fam Community Health. 2015;38(3):216-226. doi:10.1097/ FCH.0000000000000071

20. Praslova L. Adaptation of Kirkpatrick's four level model of training criteria to assessment of learning outcomes and program evaluation in higher education. Educ Assess, Eval Account. 2010;22(3):215-225. doi:10.1007/s11092-0109098-7

21. Harmon S, Rankine J, Pulcini C, Sequeria G, Collins A, Wolford J, Miller E. Trauma-informed care curriculum for pediatric residents through home visitation. Pediatrics. 2018 May, 142 (Meeting Abstract): 754.

22. Cahill T, Yunez N, Mohr D. Assessing resident confidence in screening and intervening with patients' ACE scores. STFM Resource Library. October 11, 2018. Accessed August 2, 2021. https://resourcelibrary.stfm.org/resourcelibrary/ viewdocument/assessing-resident-confidencein-s-2?CommunityKey=2751b51d-483f-45e281de-4faced0a290a\&tab=librarydocuments

23. Green BL, Saunders PA, Power E, et al. Trauma-informed medical care: CME communication training for primary care providers. Fam Med. 2015;47(1):7-14.
24. Green BL, Saunders PA, Power E, et al. Trauma-informed medical care: patient response to a primary care provider communication training. J Loss Trauma. 2016;21(2):147-159. doi:10.1080/15325024.2015.1084854

25. Bursch B, Lloyd J, Mogil C, et al. Adaptation and evaluation of military resilience skills training for pediatric residents. J Med Educ Curric Dev. 2017;4:2382120517741298. doi:10.1177/2382120517741298

26. Shamaskin-Garroway AM, Burg MM, Vasquez L, Brandt C, Haskell S. An interprofessional pilot program training medical residents in trauma-sensitive communication. J Interprof Educ Pract. 2017;8:14-19. doi:10.1016/j. xjep.2017.05.001

27. Dueweke AR, Hanson RF, Wallis E, Fanguy E, Newman C. Training Pediatric Primary Care Residents in Trauma-Informed Care: A Feasibility Trial. Clin Pediatr (Phila). 2019;58(11 12):1239-1249. doi:10.1177/0009922819859868

28. Bodendorfer V, Koball AM, Rasmussen C, Klevan J, Ramirez L, Olson-Dorff D. Implementation of the adverse childhood experiences conversation in primary care. Fam Pract. 2020;37(3):355-359. doi:10.1093/fampra/cmz065

29. Stefanski K, Mason K. Acing education: pilot curriculum on adverse childhood experiences. Med Educ. 2017;51(11):1167-1168. doi:10.1111/ medu. 13436

30. Wen FK, Miller-Cribbs JE, Coon KA, Jelley MJ, Foulks-Rodriguez KA. A simulation and video-based training program to address adverse childhood experiences. Int J Psychiatry Med. 2017;52(3):255-264. doi:10.1177/0091217417730289

31. Chokshi B, Chen KD, Beers L. Interactive case-based childhood adversity and trauma-informed care electronic modules for pediatric primary care. MedEdPORTAL. 2020;16(1):10990. doi:10.15766/mep_23748265.10990

32. Helitzer DL, Lanoue M, Wilson B, de Hernandez BU, Warner T, Roter D. A randomized controlled trial of communication training with primary care providers to improve patientcenteredness and health risk communication. Patient Educ Couns. 2011;82(1):21-29. doi:10.1016/j.pec.2010.01.021

33. Weiss D, Kassam-Adams N, Murray C, et al. Application of a framework to implement trauma-informed care throughout a pediatric health care network. J Contin Educ Health Prof. 2017;37(1):55-60. doi:10.1097/ CEH.0000000000000140

34. Sorkin DH, Rizzo S, Biegler K, et al. Novel health information technology to aid provider recognition and treatment of major depressive disorder and posttraumatic stress disorder in primary care. Med Care. 2019;57(Suppl 6 Suppl 2):S190-S196. doi:10.1097/ MLR.0000000000001036
35. Schmitz A, Light S, Barry C, Hodges K. Adverse childhood experiences and traumainformed care: an online module for pediatricians. MedEdPORTAL. 2019;15(1):10851. doi:10.15766/mep_2374-8265.10851

36. Marsicek SM, Morrison JM, Manikonda N, O'Halleran M, Spoehr-Labutta Z, Brinn M. Implementing standardized screening for adverse childhood experiences in a pediatric resident continuity clinic. Pediatr Qual Saf. 2019;4(2):e154. doi:10.1097/ pq9.0000000000000154

37. Bryant C, VanGraafeiland B. Screening for adverse childhood experiences in primary care: a quality improvement project. J Pediatr Health Care. 2020;34(2):122-127. doi:10.1016/j. pedhc.2019.09.001

38. Shamaskin-Garroway AM, McLaughlin EA, Quinn N, Buono FD. Trauma-informed primary care for medical residents. Clin Teach. 2020;17(2):200-204. doi:10.1111/tct.13082

39. Miller-Cribbs J, Bragg J, Wen F, et al. An evaluation of a simulation and video-based training program to address adverse childhood experiences. Int J Psychiatry Med. 2020;55(5):366375. doi:10.1177/0091217420951064

40. Healthy Foster Care America: Trauma Guide. American Academy of Pediatrics. 2021. Accessed September 15, 2020. https://www.aap. org/en-us/advocacy-and-policy/aap-healthinitiatives/healthy-foster-care-america/Pages/ Trauma-Guide.aspx\#foster

41. Classen CC, Clark CS. Trauma informed care. In: Cook JM, Gold SN, eds. APA Handbook of Trauma Psychology: Vol. 2, Trauma Practice. Washington, DC: American Psychological Association; 2017. 515-541. doi:10.1037/0000020025

42. Dublin S, Abramovitz R, Katz L, Layne CM. How do we get to trauma-informed practice? Retention and application of learning by practitioners trained using the core curriculum on childhood trauma. Psychol Trauma. 2020. doi:10.1037/tra0000982 